\title{
Coûts de santé: Croire que la concurrence est la panacée, c'est croire au Père Noël
}

Le système de santé suisse donne lieu à de vives préoccupations, justifiées (tout en continuant à fournir des soins de fort bonne qualité). On peine à imaginer qu'on continue à avoir longtemps des augmentations de ses coûts (reflétés dans les primes d'assurance) de l'ordre de 5 à $10 \%$ l'an. Même en sachant qu'il est inéluctable pour plusieurs raisons que ces coûts augmentent plus vite que le PIB, comme l'a rappelé plusieurs fois récemment le Ministre et professeur français J.-F. Mattei.

Pascal Couchepin va reprendre le Département fédéral de l'intérieur (DFI), dicastère des affaires sanitaires et sociales. Indépendamment d'autres considérations, il est bon qu'un magistrat dynamique y arrive avec des idées nouvelles. Il pourra secouer les relations devenues stéréotypées entre les grands partenaires concernés (OFAS, médecins, assureurs, cantons). Mais j'espère que sa perspicacité lui fera donner une attention suffisante à un espoir de Ruth Dreifuss à son départ: que la concurrence ne devienne pas la règle générale (universelle?) de la vie commune dans ce pays.

L'émulation et la compétition de bon aloi sont stimulantes et partie intégrante d'une société libérale. Mais il est impératif de voir qu'en matière de soins une concurrence agressive, comme on peut l'avoir dans d'autres secteurs, est rendue problématique dans ses effets, en particulier à cause de la difficulté majeure du patient à juger précisément de ce qu'il «achète». Elle peut avoir des effets délétères supérieurs à son bénéfice potentiel. Aujourd'hui, on voit que les assureurs cherchent pour l'essentiel à se voler des clients (si possible les bons risques), comme le font les cigarettiers qui dépensent chaque année des dizaines de millions avec l'objectif (disent-ils) de faire changer le fumeur de marque. Les impressionnants placards ou clips publicitaires des caisses-maladie me font, plus que ceux qui veulent faire acheter des voitures ou des vacances, une impression d'attrape-nigauds, d'intox. «Comme on s'en doute, la publicité est entièrement régie par le principe de persuasion. Cela fait bien longtemps qu'elle ne songe plus à argumenter rationnellement» (A. Etchegoyen, dans La Valse des éthiques). Et on espère que le nouveau chef du DFI ne cèdera pas aux chants de sirènes d'économistes théoriques adeptes du modèle des Etats-Unis, tristement incapables de soigner l'ensemble de leur population. Etats-Unis où les frais administratifs des assureurs (y compris la publicité et les procès à propos de prestations refusées) sont de l'ordre de $20 \%$ et au-delà, alors que c'est moins de $10 \%$ chez nous. C'est dire aussi qu'il serait faux d'écarter dogmatiquement l'idée d'une caisse unique.
Un problème majeur de la LAMal actuelle est qu'elle entend combiner la planification (par les pouvoirs publics) et la concurrence. Comment cela peut-il fonctionner? La planification en effet a pour but de répondre aux besoins en évitant les surdotations et les chevauchements, donc le besoin de concurrence. Choisir exclusivement la concurrence et lâcher la planification? A la mode mais dangereux: cela marchera pour les soins usuels que de nombreux médecins et hôpitaux maîtrisent; on peut espérer que les plus compétents et efficients gagneront, mais peut-être sera-ce plutôt les beaux parleurs, les jeteurs de poudre aux yeux ... De plus, il n'est guère possible dans un petit ensemble comme la Suisse d'avoir une vraie concurrence, de type commercial, dans les secteurs médicaux de pointe, universitaires. Là on n'échappe pas à une planification, idéalement courageuse et intelligente. En bref, la concurrence a sans doute une place, dont les risques pour la qualité des soins sont toutefois grossièrement sous-estimés dans certains discours néolibéraux.

Un effort principal doit être porté sur l'évaluation des prestations. Elle devrait traiter non seulement des aspects techniques médicaux ou économiques mais aussi du qualitatif: la satisfaction subjective au long cours des patients du médecin de premier recours est un paramètre qui a sa valeur. Se souvenir d'un ingrédient qui a tout de même plus d'importance en matière de soins que quand on achète de la papeterie ou des lames de rasoir, la confiance. On peut rappeler ce mot du Professeur Edouard Jequier-Doge, enseignant de policlinique médicale à Lausanne qui a beaucoup marqué ses élèves, lors de sa leçon inaugurale il y a une cinquantaine d'années, qui parlait de ces malades qui ne guérissent que par envie de faire plaisir à leur thérapeute ... Une réalité que les médecins connaissent et, à l'évidence, un facteur d'économie!

Il importe que ces évaluations soient faites de manière neutre et je comprends que nos confrères praticiens n'acceptent pas qu'elles soient du ressort des seuls assureurs. Mais on ne peut pas non plus prétendre que les assureurs, qui gèrent une partie croissante du financement, n'ont rien à dire dans l'appréciation des performances des professionnels comme du système en général. Comme toujours, la transparence et la collaboration loyale donneraient de meilleurs résultats que les combats de tranchées. Bonne chance, Monsieur Couchepin, et bonne année à tous.

Dr Jean Martin, PD, Médecin cantonal, Lausanne 